\title{
Ethological Modeling and Architecture for an Entertainment Robot
}

\author{
Ronald C. Arkin*, Masahiro Fujita**, Tsuyoshi Takagi**, Rika Hasegawa**
}

This paper presents a novel method for creating high-fidelity models of animal behavior for use in robotic systems based on a behavioral systems approach, and describes in particular how an ethological model of a domestic dog can be implemented with AIBO, the Sony entertainment robot.

\section{INTRODUCTION}

Ethology is the science of studying the behavior of animals in their natural environment. While much attention has been paid in robotics to neuroscientific models of behavior (e.g., [1,17]), less attention has been paid to realistic ethological models other than in simulated studies. It is our contention that ethology provides great insights into the design of practical robotic systems.

In this paper, a behavior systems methodology is presented drawing on work from both psychology and ethology. A specific ethological model is created for Canis Familiaris, the domestic dog. The modeling process itself is extensible to other animal species.

This model is then transformed into an ethological controller suitable for implementation on AIBO, Sony's entertainment robot (Figure 1) [7]. The underlying architecture in support of this model is discussed.

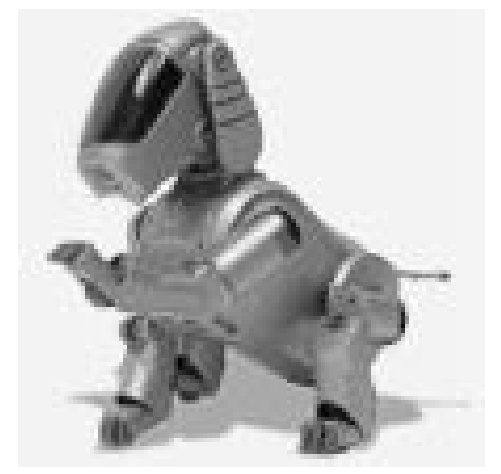

Figure 1. AIBO - Sony's Entertainment Robot

*College of Computing, Georgia Tech, Atlanta, GA, U.S.A.

**Sony Digital Creatures Laboratory, Kitashinagawa, Tokyo, Japan
The notion of robotic pets is not new. In the late 1980s, a robot pet called Petster was marketed for around $\$ 80$ U.S.D. It purred, snarled, and responded to clapping by running around. It was marketed as a mail order item apparently without much success. A more sophisticated robotic pet's construction was advocated in a book by DeCosta almost a decade earlier. Its motivation was for use in apartments that banned real dogs. It had 3 pairs of sonar sensors mounted on a rigid head. There was no control box; rather an artificial tonal language was used. It understood both the speech of the owner and used its own synthesizer to communicate. It barked for a recharge, which the owner had to provide. Neither of these systems looked more than superficially at actual canine behavior as the basis for their design.

\section{CANINE BEHAVIOR}

The domestic dog is one of the most studied mammals in existence. An extensive range of literature resources is available describing its behavior in gory detail from almost every aspect. Perhaps the two richest veins relative to the needs of a roboticist are found in the work of Scott [4] and Fox [5,12]. Scott's work in particular has led to the development of an ethogram: a categorization of all of the exhibited behaviors of the dog. This ethogram provides the basis for the model used in this work. Other ethograms $[5,18]$ for the dog exist, but Scott's is the most comprehensive.

A behavior pattern is a unique and independent piece of behavior having a complete adaptive function. The main behavioral classes for the $\operatorname{dog}$ can be characterized as shown in Table 1. The last two entries are behavioral subsystems that have been added above and beyond Scott's original ethogram but are included for implementation purposes.

\begin{tabular}{|c|}
\hline Investigative (searching/seeking) \\
\hline Sexual \\
\hline Epimeletic (care and attention giving) \\
\hline Eliminative (excretion and urination) \\
\hline Et-epimeletic (attention getting or care soliciting) \\
\hline Ingestive (food and liquids) \\
\hline Allelomimetic (doing what others in group do) \\
\hline Comfort-seeking (shelter-seeking) \\
\hline Agonistic (associated with conflict) \\
\hline Miscellaneous Motor \\
\hline Play \\
\hline Maladaptive \\
\hline
\end{tabular}

Table 1: Main Behavioral Subsystems of Dog 
Extensive research was conducted on the pertinent canine behavior literature (the literature is voluminous but [e.g., 5,8,9] are good starting points), then summarized in a series of proprietary reports for Sony Corporation.

\section{BEHAVIOR SYSTEMS APPROACH}

Clearly the organization for such a behavioral control system for the dog is a complex task. To manage this complexity, a concurrent top-down and bottom-up approach to its development has been undertaken. In particular the behavior systems approach developed by the psychologist William Timberlake [6] has provided the structure and terminology for the methods used here. Critical features of a behavior system are:

1. Motivational processes that prime other structures and help organize and maintain their sequence of expression.

2. Perceptual-motor structures (modules) that relate specific stimuli to specific responses. The response components are often sequentially and temporally related and are elicited, initiated, controlled and terminated by stimuli.

This is a functional, not physiological, model. (which is good for the purposes of robot design). Four hierarchical levels are represented using this approach:

- Systems - These are assumed to be at least partially independent from other systems. They represent a collection of motivational states that prime underlying subsystems and modules.

- Subsystems - Coherent strategies that serve the general function of the system. Activation of a subsystem sensitizes an animal to particular stimuli and potentiates responses (Figure 2).

- Modes - Motivational substates that are related to the sequential and temporal organization of action patterns with respect to terminal stimuli (e.g., Figure 3).

- Perceptual-motor modules - Respond to particular stimuli with particular response components (e.g., Figure 4).

The basic unit of output is an action-pattern: a coherent, recognizable, relatively stereotypical movement (although some variability may be present). The environment is involved in the definition of an action pattern, as well as limb and posture position and temporal patterning. Action patterns vary in strength with particular modules and may be controlled by several modules. Learning can occur through the refinement, combination and reassembling of action patterns. Of note is the fact that there is a relationship between the notion of action pattern and that of motor schema [11] as found in schema theory and utilized in previous designs in our research [10].

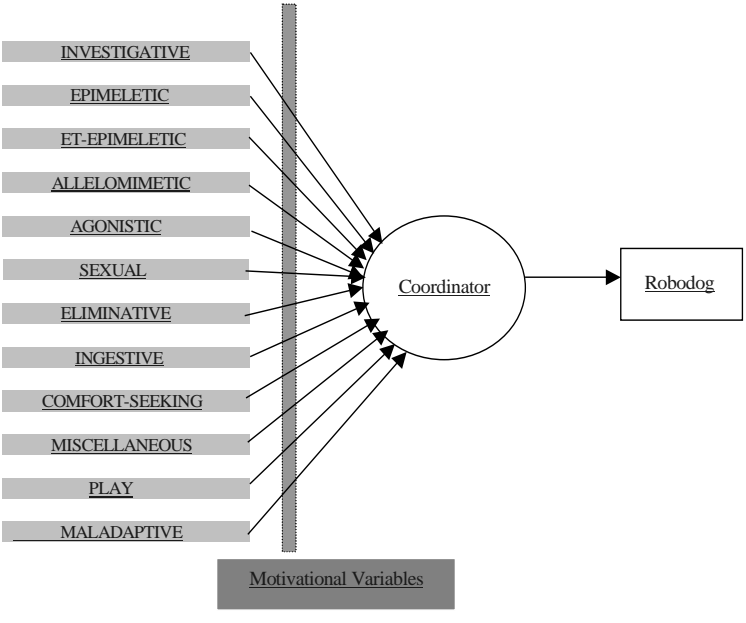

Figure 2: Complete Set of Subsystems

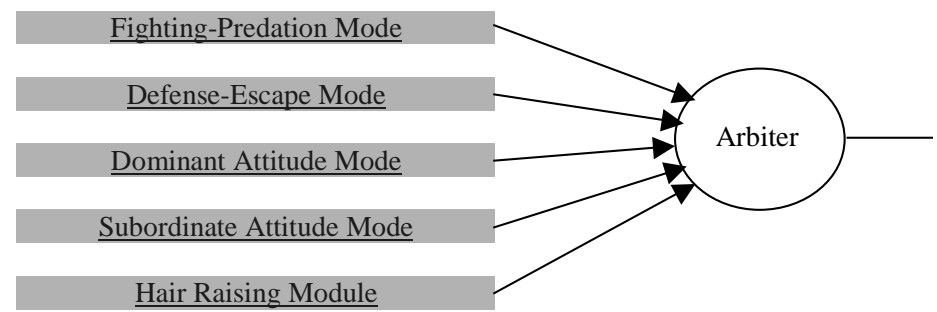

Figure 3. Modules comprising Agonistic Subsystem

Stimulus $=$ threat or dominant animal present + attack + escape route/area present + high fear

(the escape areas may include corners of rooms)

$$
\begin{aligned}
\text { Response }= & \text { run(fast, towards escape route/area }) \\
& + \text { ear-position(both, back) }
\end{aligned}
$$

Figure 4. Example: run-away module

Scott and Fuller's ethogram represents a format suitable for incremental implementation in a robotic dog. The higher-level behavioral systems are decomposed into collections of subsystems that ultimately ground into a set of robotic primitives. From the bottom-up, a collection of parameterizable robot dog primitives have been specified (taxes, reflexes, and fixed action patterns) that map onto the higher-level behavioral systems.

The advantage of this method lies in the reuse and composability of the constituent subsystems and primitives, enabling easy personalization of the robotic pet to different owners. Specifications for motivational variables that affect these components are included to allow emotional state to dynamically alter the embodied control system. 
An idealized potential input device inventory should include the following considerations in regards to fidelity for biological dogs:

- Auditory

1. Verbal communication from owner

2. Non-verbal communication from other robots/animals

3. Environmental sounds

- Visual

1. Displays of owner

2. Displays of other animals/robots

3. Specific behavioral cues (colors, shapes, etc.)

- Tactile

1. Petting

2. Collision

3. Holding

- $\quad$ Force - to detect restricted movement

- Olfactory

- Dermal (temperature) Taste

- Vestibular

Not all of these are currently available on AIBO.

A preliminary motor output set was developed to enable a mapping of the behaviors onto the actuators. This list of output primitives serves as the set of response functions for the developed behaviors [11]. The list is too extensive to reproduce here, but includes sets of actions organized under major classes of actuation such as tail, head, legs, body, vocalization, locomotion, and elimination.

Two of the smaller behavioral subsystems are reproduced with their behavioral modules listed in Figure 5. For all 12 subsystems there are over 140 total behavioral modules.

\begin{tabular}{|ll|}
\hline - & Investigative \\
- & Head in air sniffing module \\
- & Sniffing conspecific module \\
- & Nort looking module \\
- & Scent tracking module \\
- & Encounter module \\
- & Pointing module \\
- & $\quad$ Et-epimeletic \\
- & Yelping for attention module \\
- & Licking face/hands module \\
- & Pawing module \\
- Jumping up module \\
- & Pup rooting for nipple module \\
- & Close following of caregiver module \\
\hline
\end{tabular}

Figure 5. Behavior modules within two of the 12 major subsystems
The following motivational variables were recommended for eventual implementation based on literature studies of canine emotionality and motivation (e.g., [19]):

\begin{tabular}{|c|c|}
\hline Guilt & Frustration \\
Boredom & Anger/aggression \\
Fear & Patience \\
Submission / dominance & Spite \\
Happiness/love & Sadness/depression \\
Jealousy (pack competition) & Tense/relaxed \\
Alertness & Sexual \\
Hunger/thirst & Fatigue \\
Pain & Gregariousness \\
Elimination needs & Loyalty \\
Temperature & Somnolence \\
& \\
\hline
\end{tabular}

Table 2. Motivational Variables

\section{COORDINATION FUNCTIONS}

Numerous approaches regarding choice of the coordination functions for the behaviors were investigated. While it was determined that a motivational space approach [20] would likely be the best in the long-term, it was decided that using a model developed initially by Ludlow $[15,16]$ to model biological action-selection and then later imported to computer graphics applications by Blumberg $[13,14]$ would serve well in the short-term.

Ludlow $[15,16]$ introduced the notions of lateral inhibition and the fatiguing of behaviors later utilized by others in the action-selection community. Blumberg's Hamsterdam project [15] speaks to persistence, avoiding behavioral dithering (the rapid oscillation between different behaviors) through the incorporation of lateral inhibition and fatigue. It also incorporates time-sharing for low priority behaviors to execute in the presence of a high priority behavior. The coordination mechanism is a winnertake-all approach that incorporates both releasing mechanisms (external stimuli) and endogeneous variables (internal data). It can model ethologically observed motivational isoclines. Strict homeostatic modeling is not required however. Blumberg's later work extended this model into the ALIVE system and was based on a dog-like graphical character [14]. Much of his work is drawn from the animation community, but very little from or to robotic systems functioning in the real world.

The overall action-selection mechanism is summarized as follows:

1. At the top-level behavior group competition occurs by updating releasing mechanism values and combining the result with the motivation and interest levels. Inhibition is then applied from other competing behaviors. This process is iteratively repeated until only one behavior has a non-zero value. This is the active behavior within that group. 
2. Behaviors within the selected group that are not active can issue secondary commands (i.e., they can execute if it doesn't cause a problem with the selected primary behavior).

3. The process is recursive through the hierarchy until a single behavior is selected to issue the motor commands.

A variant of this approach has been used for initial implementation on AIBO, where a recursive descent through the behavioral systems hierarchy, as described in the previous Section, occurs until one single behavior is selected for execution.

\section{IMPLEMENTATION}

In order to verify the advantages of the ethological approach, we implemented the model described in the previous sections. We focus on checking if the following advantages hold in the actual robotic implementation.

(1) The fusion of internal motivations and external stimuli

(2) The coordination of behaviors via lateral inhibition

(3) Computational efficiency with a layered architecture

In order to simplify and shorten development time, we implemented a subset of the whole model with limited perception (recognition targets) as follows:

(1) Only 3 subsystems shown in Fig. 8 are realized in part

(2) Only 3 objects, WATER, FOOD, and MASTER, can be recognized by color classification

Fig.6 shows the implemented software architecture on the AIBO. As described in the previous sections, roughly speaking, there are 3 major parts, Release Mechanism, Motivation Creator, and Action Selection Module.

The Release Mechanism component computes its output RM[I] (see Fig. 7) using environmental perceptual results such as the distance to a recognized object. As itemized above, we only use the color camera signal for this purpose and only 3 objects can currently be recognized.

Motivation Creator computes its output Mo[I] (see Fig. 7) using an Instinct and Emotional Model, which has 6 internal variables: nourishment, moisture, bladder, tiredness, curiosity, and affection. Furthermore, another 6 variables act to keep the 6 internal variables within some bounded values. These are called instinct variables, which include hunger, thirst, eliminate, tiredness, curiosity, and affection. The output of the Motivation Creator Mot[I] is computed using these instinct variables.

In the Action Selection Module, a behavior variable V[I] is computed using a function of RM[I] and Mot[I] as shown in the graph of Fig.7. The computation is carried out from behaviors in the higher layer. The lateral inhibition to avoid the behavioral dithering described in the previous section is also carried out here so that the system can select one behavior. From the highest layer (subsystems) to the lowest layer (primitive modules), the computations are performed to select a proper action command that is sent to a FiniteState-Machine where the specific sequences on how to complete the command are described.

Thus, the action to be executed is selected based on the value $\mathrm{V}[\mathrm{I}]$, which is affected by both Mot $[\mathrm{I}]$ related to the internal variables and $\mathrm{RM}[\mathrm{I}]$ related to the external stimuli. For example, even if the robot has high motivation for ingestive behavior, without the relevant external stimuli, then the robot doesn't select the ingestive behavior, and vice versa.

Figure 8 shows a layered and tree structured architecture for subsystems, modes, and primitive modules. Figure 9 shows the implemented behavior tree, where 3 subsystems, investigative, ingestive, and play, are implemented. Investigative means investigative behaviors such as walk around (locomotion), ingestive means ingestive behaviors such as eating or drinking, and play means interactive behaviors with a human such as giving a paw.

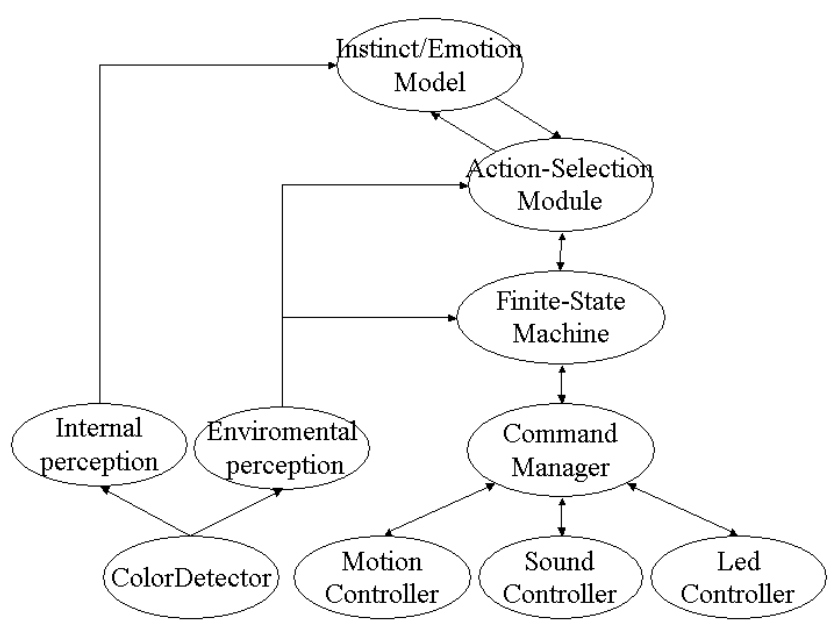

Figure 6. Software architecture

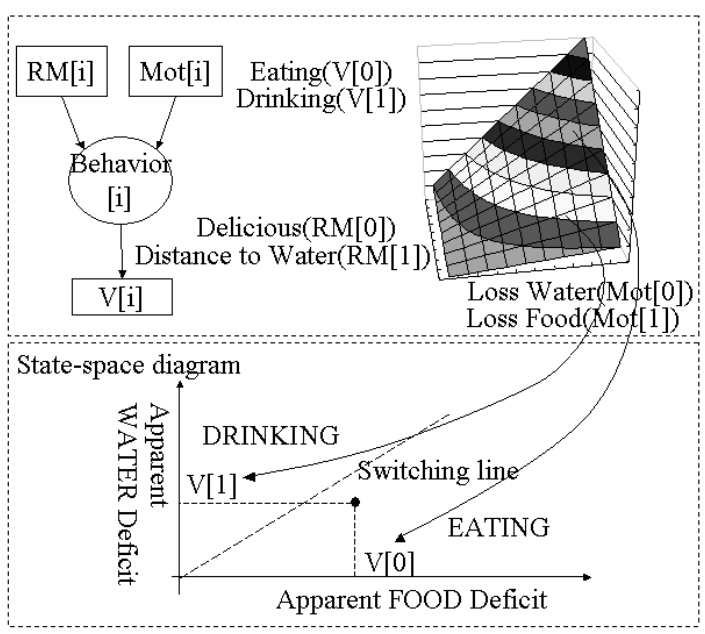

Figure 7. State-space Diagram 


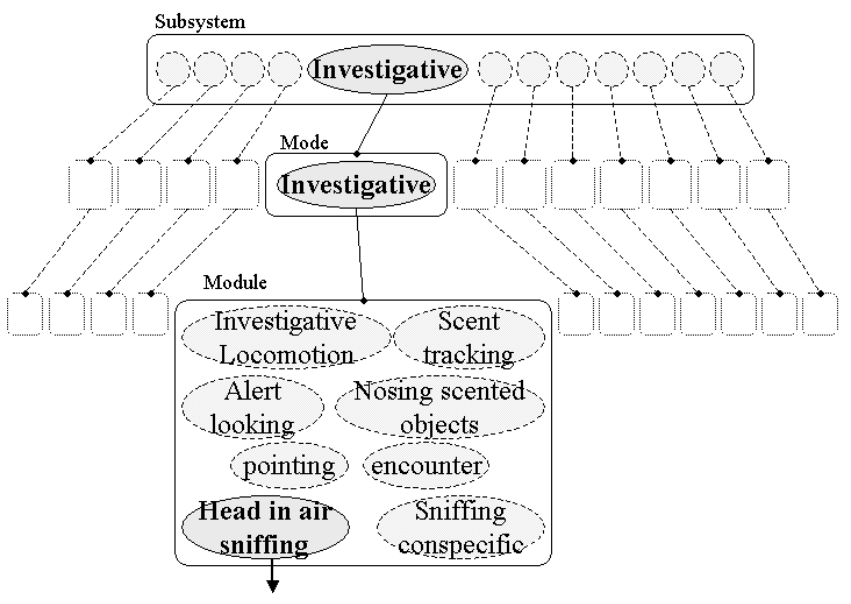

Figure 8. Behavioral Tree (Whole)

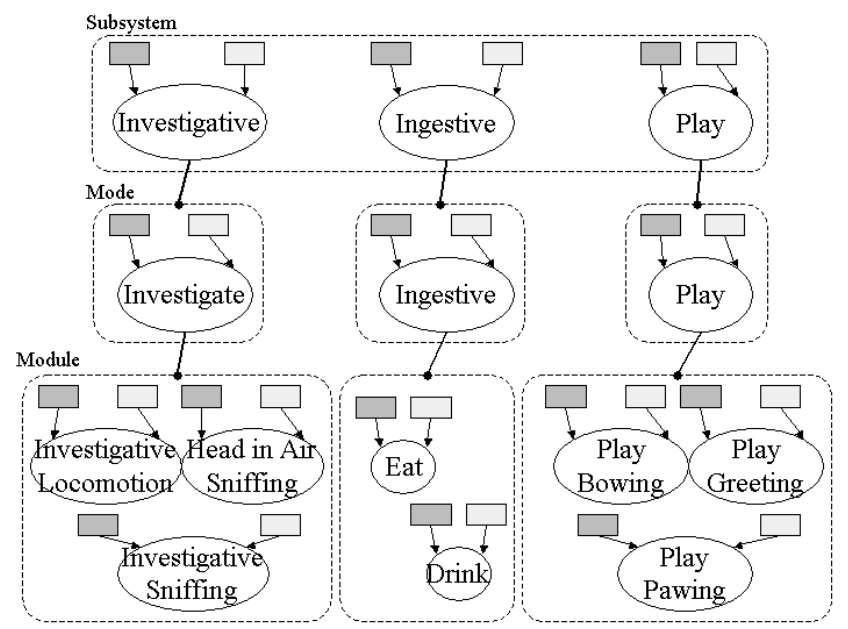

Figure 9. Behavioral Tree (Implemented)

\section{EXPERIMENTS AND RESULTS}

In order to verify if the advantages of this approach are achieved, we build a test field as shown in Fig. 10. For easy recognition, we make red, blue, and green circles with 12$\mathrm{cm}$ diameter, which correspond to FOOD, WATER, and MASTER respectively. The field is $120 \mathrm{~cm}$ square and is surrounded by walls. We placed the robot described in the previous section in the field and measure the RM[I], Mot[I], $\mathrm{V}[\mathrm{I}]$, selected behavior, and so on.

Figures 11-15 show various time sequences of some relevant measurements. Figure 11 shows the Time-Instinct variable graph. Figure 12 and 13 show Time-Motivation variable graphs corresponding to Mot[I] of subsystems and modules. Figure 14 shows a Time-Release Mechanism $(\mathrm{RM}[\mathrm{I}])$ variable graph, and Figure 15 shows the time sequence of selected behaviors. Here, the 6 internal variables are decreased as time passes but increased while the corresponding behavior is executed.

Comparing Figure 11 with Figure 15, we can observe an increase of the instinct variables as well as its decrease when the corresponding action is selected. Moreover, comparing Figures 12 and 13 with Figure 15, we can observe that the corresponding action is not selected (as expected) even when higher Motivation variable Mot[I] is found in some time periods. Then, comparing between Figures 14 and 15, in this period, the Release Mechanism value $\mathrm{RM}[\mathrm{I}]$ is small, so not enough external stimuli is presented within that period.

During such a period, the system selected "investigative" behavior. Thus, the motivation variables or the internal variables and the external stimuli affect the action selection mechanism in this system, as anticipated.

We encountered a problem, when an action cannot be selected properly. For example, when "hunger" motivation is large, and WATER exists, then the highest layer selects "ingestive" behavior. Because WATER doesn't produce a big Release Mechanism value for the eating behavior, there is no action that has both of larger RM[I] and Mot[I] in the lowest layer of the selected ingestive subsystem. This can be avoided by designing a proper tree structure.

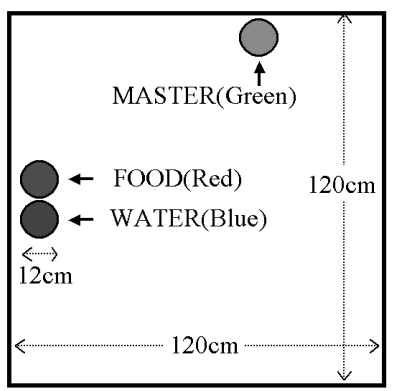

Figure 10. Field

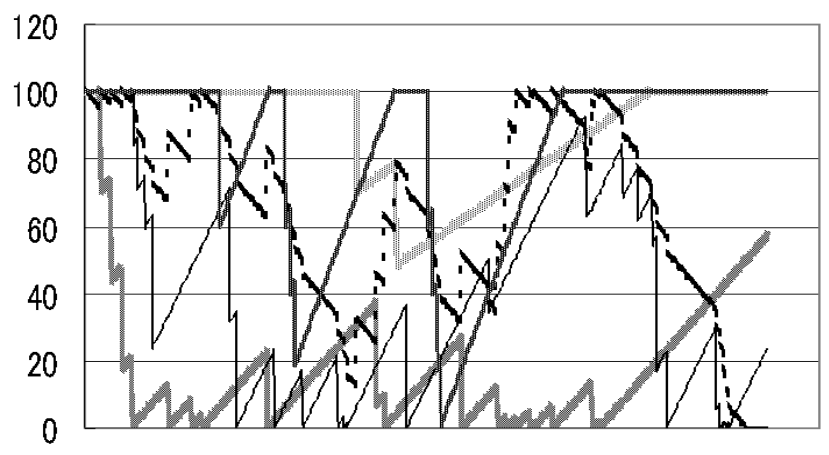

Figure 11. Instinct-Time graph

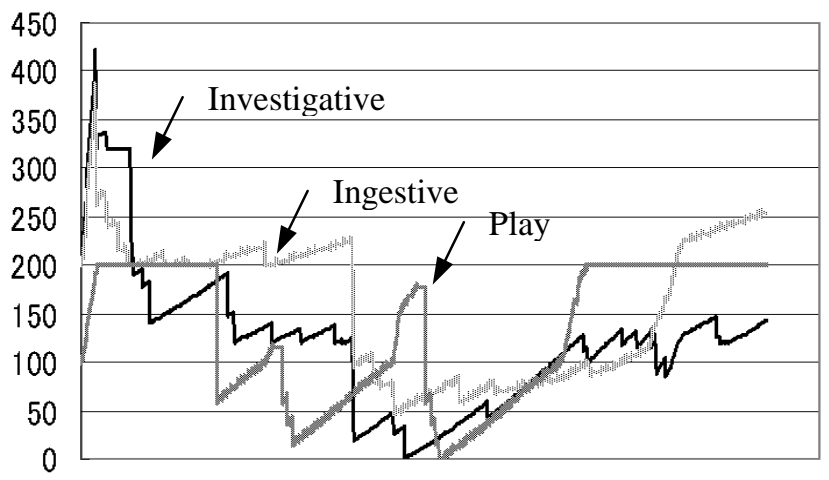

Figure 12. Motivation-Time graph for subsystem 


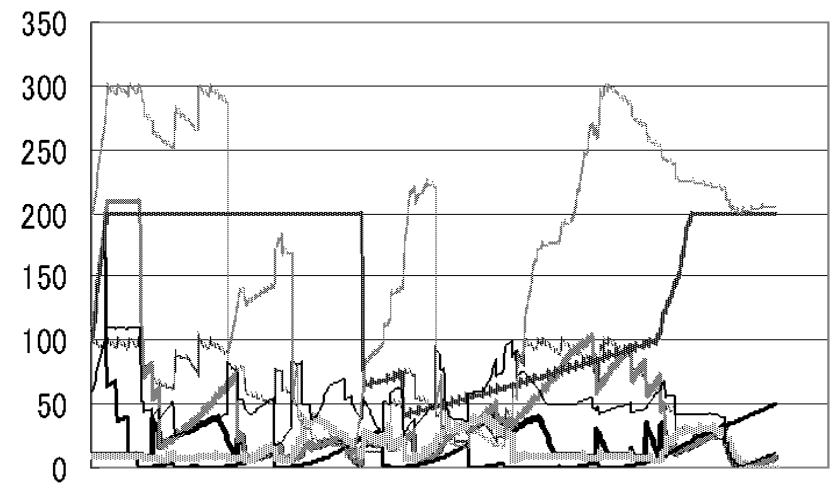

Figure 13. Motivation-Time graph for Module

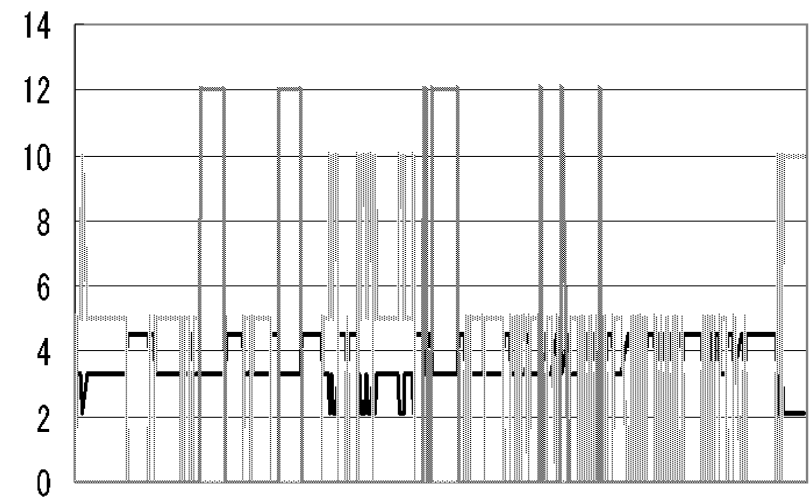

Figure 14. Release Mechanism-Time graph

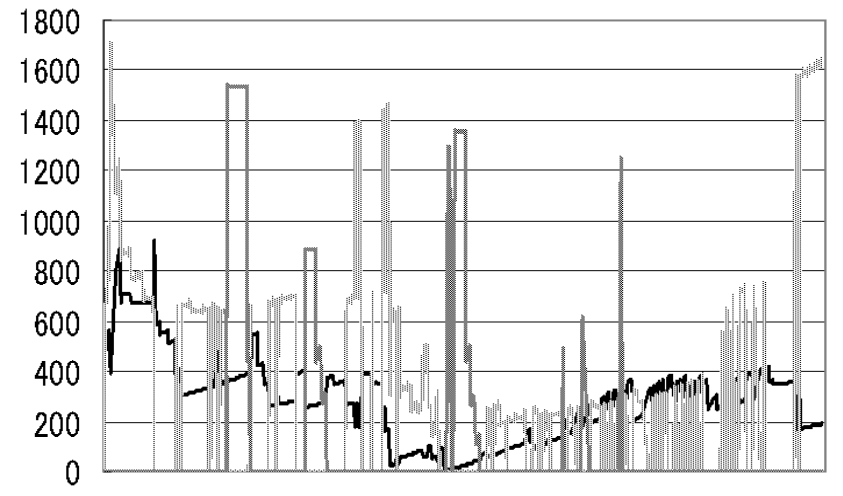

Figure 15. Behavior-Time graph

\section{SUMMARY AND CONCLUSIONS}

An ethological controller derived directly from canine behavior has been developed for use in Sony's AIBO. It employs the behavioral systems approach as developed by Timberlake [6] as applied to Scott's ethogram of canine behavior [4]. In the short term, behavioral coordination is accomplished through a variant of the Ludlow [15]Blumberg [14] action-selection mechanism.

This model has been successfully implemented in part on AIBO. We implemented 3 subsystems with 8 primitive actions with this model. The complex action selection mechanism is observed, which is caused by both internal variables and external stimuli. Moreover, efficient computation is performed by the layered, tree-structured architecture.

\section{References}

[1] Beer, R., Intelligence as Adaptive Behavior: An Experiment in Computational Neuroethology, Academic Press, N.Y., 1990.

[3] DeCosta, F., How to Build Your Own Working Robot Pet, Tab Books, Blue Ridge Summit, PA, 1979.

[2] Engelberger, J.F., Robotics in Service, MIT Press, 1989.

[4] Scott, J.P. and Fuller, J.L., Genetics and the Social Behavior of the Dog, University of Chicago Press, Chicago, IL, 1965.

[5] Fox, M., The Dog: Its Domestication and Behavior, Garland, New York, 1978.

[6] Timberlake, W. and Lucas, G., "Behavior Systems and Learning: From Misbehavior to General Principles", in Contemporary Learning Theories: Instrumental Conditioning Theory and the Impacts of Biological Constraints on Learning, S. Klein and R. Mowrer (eds.), LEA Associates, Hillsdale, NJ, 1989.

[7] Sony Robot Reference User's Manual.

[8] Coren, S., The Intelligence of Dogs, Bantam, New York, 1994. [9] Buytendijk, F., The Mind of the Dog, Arno Press, New York, 1973.

[10] Arkin, R.C., "Motor Schema-based Mobile Robot Navigation", International Journal of Robot Research, Vol. 8, No. 4, pp. 92-112.

[11] Arkin, R.C., Behavior-based Robotics, MIT Press, 1998.

[12] Fox, M., Integrative Behavior of Brain and Behavior in the Dog, University of Chicago Press, Chicago, 1971.

[13] Blumberg, B., "Action-Selection in Hamsterdam: Lessons from Ethology", From Animals to Animats 3, ed. Cliff et al, MIT Press, 1994, pp. 108-117.

[14] Blumberg, B., Old Tricks, New Dogs: Ethology and Interactive Creatures, Ph.D. Thesis, MIT Media Lab, Massachusetts Institute of Technology, Cambridge, MA 1996.

[15] Ludlow, A., "The Behaviour of a Model Animal", Behaviour, LVIII, 1-2, pp. 131-172, 1976.

[16] Ludlow, A., "The Evolution and Simulation of a Decisionmaker", in Analysis of Motivational Processes, F. Toates, and T. Halliday, Academic Press, New York, 1980.

[17] Touretzky, D. and Saksida, L., "Operant Conditioning in Skinnerbots", Adaptive Behavior, Vol. 5, No. 3-4, 219-247, 1997.

[18] Serpell, J. (Ed.), The Domestic Dog: Its Evolution, Behaviour, and Interactions with People, Cambridge University Press, 1995.

[19] Milani, M., The Body Language and Emotion of Dogs, Quill, New York, 1986.

[20] McFarland, D. (ed.), Motivational Control Systems Analysis, Academic Press, London, 1974.

[21] Timberlake, W. and Lucas, G., 1989, Behavior Systems and Learning: From Misbehavior to General Principles, in Contemporary Learning Theories: Instrumental Conditioning Theory and the Impact of Biological Constraints, ed. Klein S. \& Mowrer, R. Lawrence Erlbaum Associates, Inc. Hillsdale NJ.

[22] McFarland, D. \& Sibley, R., 1975, The behavioral final common path, Philosophical Transactions of the Royal Society, B. 270 . 